\title{
Sken\&agraphie
}

SKIINagraphil Coulisses des arts du spectacle et des scènes

émergentes

5 | 2018

Juste la fin du monde, de Lagarce à Dolan

\section{Luca Ronconi metteur en scène de Lagarce : la langue contre l'image}

\section{Ester Fuoco}

\section{OpenEdition \\ Journals}

Édition électronique

URL : http://journals.openedition.org/skenegraphie/1525

DOI : 10.4000/skenegraphie.1525

ISSN : 2553-1875

\section{Éditeur}

Presses universitaires de Franche-Comté

\section{Édition imprimée}

Date de publication : 1 janvier 2018

Pagination : 169-175

ISBN : 978-2-84867-5609-8

ISSN : $1150-594 X$

\section{Référence électronique}

Ester Fuoco, «Luca Ronconi metteur en scène de Lagarce : la langue contre l'image », Sken\&agraphie [En ligne], 5 | 2018, mis en ligne le 01 janvier 2019, consulté le 30 avril 2019. URL : http:// journals.openedition.org/skenegraphie/1525; DOI : 10.4000/skenegraphie.1525

Ce document a été généré automatiquement le 30 avril 2019

Presses universitaires de Franche-Comté 


\title{
Luca Ronconi metteur en scène de Lagarce : la langue contre l'image
}

\author{
Ester Fuoco
}

1 Après d'excellentes traductions et mises en espace de Barbara Nativi ${ }^{1}$, réservées à un public restreint, c'est en 2009 que l'Italie a découvert Jean-Luc Lagarce avec Juste la fin du monde et Les Prétendants, mis en scène au Piccolo Teatro de Milan par son directeur artistique, Luca Ronconi ${ }^{2}$. Depuis, rares ont été les reprises de Juste la fin du monde en Italie.

2 Lagarce et Ronconi ont consacré leur vie au théâtre. Acteur sensible et doué, Ronconi a été dirigé par tous les monstres sacrés du théâtre italien depuis ses vingt ans, bien qu'il ait admis avoir embrassé cette carrière sans trop de conviction. Cet «enfant gâté du théâtre italien » est devenu un jour " le metteur en scène de la folie »" Les deux hommes ont donné pendant toute leur carrière une attention particulière au public, sans chercher à le flatter. «J'aime bien que le public soit content. Pas tout le temps [...]. On ne doit pas oublier que tout est faux. Le théâtre est un jouet ${ }^{4}$. Ces deux metteurs en scène ont fait des spectacles mettant au centre le texte, la langue, les mots. Ils attribuent aux mots leur exacte valeur, autour des énoncés performatifs des personnages. Tous les deux s'appuient sur un projet dramaturgique développé : travail du texte avec un stylo, étude des références et du contexte, constitution d'une documentation et collecte d'images, réalisation de notes pour les techniciens, les costumières et les acteurs ${ }^{5}$.

\section{Les raisons d'une rencontre}

3 Pendant sa longue carrière, Ronconi a monté très peu d'auteurs contemporains. Il a reconnu avoir choisi ce texte sans ignorer la difficulté pour les spectateurs qui ne connaîtraient ni l'auteur, ni sa langue. On y parle beaucoup, dit Ronconi, mais avec réticence ${ }^{6}$. Il avait beaucoup apprécié la mise en scène de Michel Raskine donnée à la Comédie-Française en 2008.

Ronconi exige des acteurs qu'ils travaillent sur eux-mêmes et non sur l'idée qu'ils se font d'eux-mêmes. Le processus est difficile et dangereux ; c'est pourquoi il a aimé mettre en 
scène Lagarce, lui qui ne construit pas de personnages mais donne à voir l'inconscient. Depuis ses débuts, une des spécificités du travail de Ronconi a été la volonté de mettre le spectateur dans la position d'arbitre face à une dramaturgie ouverte. Parce que cet objectif a été mal perçu, il a été nommé le metteur en scène de la folie. Ce qui caractérise l'esthétique de ces deux artistes est une forme de jeu dramaturgique brechtien, avec une rupture de la mimesis et une fragmentation du texte, souvent dépourvu d'une conclusion précise ou conçu pour se poursuivre au-delà de la fin de la représentation.

Par ailleurs, avec ses acteurs, Ronconi construit un travail sur le langage méticuleux, presque obsessionnel. Dans un entretien, Ronconi a parlé de son désir de mettre en scène Juste la fin du monde comme d'un défi. Il était attiré par le thème de l'œuvre et la parole théâtrale particulière du dramaturge français qui se révèle assez proche de la langue parlée en Italie. Ce qu'il a remarqué chez Lagarce, c'est l'écart qui existe entre la pensée et son expression langagière, avec les blocages dans la communication. Cet interstice entre ce que l'on veut dire et ce que l'on dit est présent dans la conscience du locuteur italien. Cependant, cette mise en scène a été différente de ses travaux habituels. Elle était plutôt statique, sans scénographie spectaculaire, le jeu des acteurs reposant sur une violence intériorisée. Ronconi a également évoqué des motivations très intimes : il était malade et, contrairement à Louis, il a, depuis toujours, été défini comme l'homme sans famille ${ }^{7}$.

\section{Le logocentrisme malgré tout}

Ronconi connait par cœur les textes qu'il choisit et il accomplit une sorte d'examen psycho-analytique de chaque phrase, en cherchant ainsi sa vérité profonde dont l'auteur n'avait peut-être pas pleinement conscience en écrivant. Il donne à chaque acteur des indications très précises et rigoureuses que chacun adapte à son tempérament et à sa personnalité. Il a utilisé la traduction de Franco Quadri, pleine de fines nuances. Elle assure la qualité de la représentation du langage sur le plateau. Quadri a fait une traduction presque littérale pour respecter l'oralité du texte qui se déploie ; sans perdre le lien entre la parole et le sens auquel elle se réfère, il a su décalquer la maladresse du dire lagarcienne.

7 Comme il s'agit de deux langues différentes, le traducteur aurait pu choisir de couper suivant le rythme de la langue italienne. Pourtant, il a combiné le respect du prototexte avec des retours à la ligne qui ne suivent pas ceux de Lagarce, trahissant ainsi la géographie harmonique du texte originel. Il abandonne l'effet du verset français et change les césures structurant la pensée, donc le souffle, des personnages. Par conséquent, Ronconi, en travaillant avec ses acteurs, a modifié la temporalité du jeu, les pauses et les accents prévus par le texte de Lagarce en cherchant à sauver le sens - et non le son - des mots du texte. Contre l'idée proposée par le personnage, les acteurs privilégient celle donnée par le metteur en scène pour s'approprier le texte.

\section{La genèse du spectacle}

8 Le travail à la table avec les comédiens et l'équipe du spectacle occupe normalement 10-15 jours. Dans le cas présent, il a été porté à 25 jours. C'est le metteur en scène italien qui, conformément à la tradition, lit et joue tous les personnages les premiers jours. Par 
certains côtés, Ronconi enseigne à ses acteurs avant tout la manière de lire un texte. Il ouvre le livre à n'importe quel endroit pendant les premières répétitions et se focalise sur des moments forts, sans forcément suivre l'ordre du récit ${ }^{8}$. La première étape est la décomposition du texte dans ses unités signifiantes : mots, syntagmes, phrases... Chaque réplique est décortiquée et observée à la loupe, à la recherche de toutes les parcelles possibles de sens qui devront émerger à travers la voix et le corps de l'acteur. Il s'agit d'une gymnastique philologique où le sens devient corps, prend corps. Si un écart émerge entre les intentions attribuées au texte et la restitution de la part de l'acteur, le metteur en scène reprend et corrige patiemment. De cet exercice sur le sens du texte découle vraiment le rythme du discours en scène.

9 Après ce travail d'explication du sens, des références et des difficultés de l'œuvre, les acteurs commencent à jouer leur rôle pendant plusieurs répétitions avec l'aide d'un enregistreur. Puis vient le travail sur la gestuelle des acteurs. Ronconi part toujours de l'immobilité avant de trouver le geste, tout comme du silence avant de faire éclore le verbe. Mais il n'y a pas seulement l'énergie que l'acteur doit donner au texte; il y a aussi le rapport entre les personnages, dont découlent l'occupation et la répartition de l'espace scénique. Cette occupation doit être dynamique, faite de positions et de déplacements qui, au-delà de préoccupations naturalistes, doivent exprimer les rapports symboliques entre personnages. Ronconi privilégie des géométries rigoureuses : la scène est toujours parcourue comme une grille imaginaire, incluant des diagonales et des circonférences. Ensuite, l'éclairage et le décor sont prêts seulement quinze jours avant le spectacle.

La méthode de travail de Ronconi est celle du "bottom-up" : il ne part jamais d'une idée préconstruite $\mathrm{du}$ texte, d'une clé interprétative unique à laquelle ramener l'interprétation entière de la pièce, à l'inverse de la méthode "top-down" qui avait caractérisé la première histoire de la mise en scène. Une telle approche est certainement plus en accord avec la mentalité post-moderne qui hérite des outils de l'âge du soupçon, aiguisés par les découvertes de Marx, de Freud ou du structuralisme linguistique et anthropologique. Cependant, c'est une méthode qui risque d'éclipser la dimension émotive des personnages, avec un abaissement de la tension dramatique sous le poids de cette puissante machine herméneutique.

\section{La mise en scène}

11 La mise en scène a été caractérisée par une simplicité grandiose de sorte que l'on a rendu visible et lisible le plus petit détail de la pièce, dans une perspective brechtienne ${ }^{9}$, si l'on entend par là l'« agencement, dans un certain ordre, d'éléments séparés, relativement autonomes, au service d'une fin déterminée $»^{10}$. Le démantèlement intentionnel des rouages traditionnels du théâtre fait que le récit et la fable ne se construisent plus en vertu de la nécessité de la vraisemblance. Le décor a été créé par Marco Rossi. Il se construit sur deux niveaux, avec très peu d'objets en scène : des chaises, un fauteuil, une petite table, des entrées et des sorties latérales, en position de rehaussement par rapport à l'avant-scène. Ronconi se débarrasse de tous les éléments anecdotiques de la scénographie. L'espace, presque vide, est coloré par différentes tonalités de gris. Au premier plan, il présente une sorte de purgatoire qui est réservé à Louis. Celui-ci voit tout le monde sur le plateau et il se sent vu par tous. Lorsque le rideau se lève, le spectateur est face à un théâtre de la mémoire, ou du rêve. 

blanche, comme celle d'un hôpital ${ }^{11}$, sculpte les éléments de la représentation et module l'espace en lui conférant une impression surréelle même si l'on se retrouve à l'intérieur d'une maison. Le travail de Ronconi sur l'espace n'est pas la conséquence d'un maniérisme mais d'une recherche d'organicité et de cohérence. contemple déjà comme s'il était de l'autre côté du miroir. L'espace, épuré à l'extrême, découpé par un cadre de scène noir, laisse voir les personnages comme sur une photo sépia: c'est comme si la lumière crue du souvenir tentait de les fixer avant leur disparition. Cet espace vide est nécessaire pour le fonctionnement herméneutique propre à un théâtre complexe, un théâtre de la non-représentation, un théâtre qui se constitue en quelque sorte en simulacre, simulant jusqu'à la présence réelle des acteurs sur la scène.

Ronconi place les acteurs dans l'espace réduit de la maison, sédentaires, incapables de tout mouvement. Le huis-clos lagarcien produit un effet de claustration. La tragédie moderne, liée à l'idée d'inanité de la vie ${ }^{12}$, s'exprime à travers une distribution symbolique des espaces intimes de la maison: l'espace des dominants et celui des dominés. Ainsi, Catherine est toujours éloignée de la famille ou derrière Antoine. Le haut s'oppose au bas: Suzanne, sœur plus jeune, est souvent accroupie, assise par terre, avec ses deux frères lointains.

15 Les cinq acteurs ${ }^{13}$ jouent totalement à l'italienne, avec des sentiments très incarnés, à rebours de la petite musique lagarcienne. La récitation demandée par le metteur en scène est toujours accentuée afin de caractériser la névrose qui touche son apogée dans le rôle de la Mère : elle ne regarde jamais Louis, laconique. Antoine semble être le plus rationnel. Suzanne, en parlant de ses souvenirs, délire. Le sentiment de culpabilité les détruit tous. La diction nette et scrupuleuse rend sensible, par ses traits artificiels, le drame familial.

Cette mise en scène de Ronconi, s'oppose à l'idée d'un théâtre qui construirait des images du réel ; c'est un univers de présences qui font sens, contre le théâtre traditionnel. «Il faut défendre la langue contre l'image, nous vivons l'absence de la langue. Dans l'absence du verbe [...]. Je ne suis pas contre le travail corporel, cela me parait même essentiel, mais lui aussi doit être soutenu par un discours ${ }^{14} »$. Ronconi donne corps à des voix et rend vides des corps qui se montrent comme des esprits, respectant l'ethos du personnage lagarcien et sa façon de se tenir sur le seuil.

\section{NOTES}

1. Directrice artistique du théatre de recherche Teatro della Limonaia de Florence.

2. Luca Ronconi, né en 1933, est considéré comme l'un des plus importants et influents directeurs de théâtre en Europe. Il s'est fait connaître du public français par sa mise en scène d' Orlando furioso d'après l'Arioste dans les Halles de Baltard à Paris en 1970. Il dirige le Teatro Stabile de Turin pendant 7 ans et, en 1998, à la suite du décès de Giorgio Strehler, il devient 
directeur artistique du Piccolo Teatro où il dirige également l'école d'art dramatique fondée par Paolo Grassi et Giorgio Strehler.

3. Franco QUADRI, Luca Ronconi, ou le rite perdu, traduit de l'italien par Ornella Volta, Paris, Union Générale d'Éditions, «10/18 », 1974, p. 12.

4. «Entretien par Jean-Michel Potiron », 1994, in Jean-Luc LAGARCE, Mes projets de mises en scène, Besançon, Les Solitaires intempestifs, 2014, p. 17.

5. Voir ibid., p. 20.

6. Traduction d'un entretien disponible sur youtube: https://www.youtube.com/watch? v=IMKjpgURVEg

7. Il a simplement grandi et vécu avec sa mère.

8. Voir Italo moscato, Luca Ronconi. Un grande maestro negli anni dei guru, Rome, Ediesse, « Arte e Lavoro ", 2016, p. 11.

9. Il y avait ainsi des enseignes lumineuses pour indiquer chaque étape du spectacle : prologue, parties, épilogue.

10. Voir Marie SilhouetTe \& Jean-Marie VAlentin (dir.), Bertolt Brecht, La théorie dramatique, Paris, Klincksieck, « Germanistique », 2012, p. 61.

11. Il faut savoir que Ronconi a mis en scène ce texte alors qu'il connaissait de sérieux problèmes de santé.

12. Voir Jean-Pierre SARRAZAC, La Parabole ou l'enfance du théâtre, Belfort, Circé, 2002, p. 163.

13. Riccardo Bini (Louis), Melania Giglio (Suzanne), Pierluigi Corallo (Antoine), Francesca Ciocchetti (Catherine), Bruna Rossi (La Mère).

14. «Entretien avec Jean-Michel Potiron », 1994, in Jean-Luc LAGARCE, Mes projets de mises en scène, op. cit., p. 26.

\section{INDEX}

Mots-clés : Jean-Luc Lagarce, Juste la fin du monde, Luca Ronconi, théâtre et traduction

\section{AUTEUR}

\section{ESTER FUOCO}

Ester Fuoco est actuellement doctorante en Digital Humanities - Arts et Spectacle à l'Université de Gênes, en cotutelle avec l'Université Paris Diderot. Ses recherches concernent le théâtre des $\mathrm{XX}^{\mathrm{e}}$ et $\mathrm{XXI}^{\mathrm{e}}$ siècles, avec un intérêt particulier pour l'application et l'utilisation des nouvelles technologies dans le domaine de la performance. Elle a collaboré en 2014 avec la chaire d'Esthétique du Spectacle à l'Université de Milan et en 2015 comme enseignante à l'Académie des Beaux Arts de Milan. 